\title{
Rapid Development of Morphological Descriptions for Full Language Processing Systems
}

\author{
David Carter \\ SRI International Cambridge Computer Science Research Centre \\ 23 Millers Yard, Mill Lane \\ Cambridge CB2 1RQ, U.K. \\ dmc@cam.sri.com
}

\begin{abstract}
I describe a compiler and development environment for feature-augmented twolevel morphology rules integrated into a full NLP system. The compiler is optimized for a class of languages including many or most European ones, and for rapid development and debugging of descriptions of new languages. The key design decision is to compose morphophonological and morphosyntactic information, but not the lexicon, when compiling the description. This results in typical compilation times of about a minute, and has allowed a reasonably full, feature-based description of French inflectional morphology to be developed in about a month by a linguist new to the system.
\end{abstract}

\section{Introduction}

The paradigm of two-level morphology (Koskenniemi, 1983) has become popular for handling word formation phenomena in a variety of languages. The original formulation has been extended to allow morphotactic constraints to be expressed by feature specification (Trost, 1990; Alshawi et al, 1991) rather than Koskenniemi's less perspicuous device of continuation classes. Methods for the automatic compilation of rules from a notation convenient for the rule-writer into finitestate automata have also been developed, allowing the efficient analysis and synthesis of word forms. The automata may be derived from the rules alone (Trost, 1990), or involve composition with the lexicon (Karttunen, Kaplan and Zaenen, 1992).

However, there is often a trade-off between runtime efficiency and factors important for rapid and accurate system development, such as perspicuity of notation, ease of debugging, speed of compilation and the size of its output, and the independence of the morphological and lexical components. In compilation, one may compose any or all of (a) the two-level rule set,

(b) the set of affixes and their allowed combinations, and

(c) the lexicon;

see Kaplan and Kay (1994) for an exposition of the mathematical basis. The type of compilation appropriate for rapid development and acceptable run-time performance depends on, at least, the nature of the language being described and the number of base forms in the lexicon; that is, on the position in the three-dimensional space defined by (a), (b) and (c).

For example, English inflectional morphology is relatively simple; dimensions (a) and (b) are fairly small, so if (c), the lexicon, is known in advance and is of manageable size, then the entire task of morphological analysis can be carried out at compile time, producing a list of analysed word forms which need only be looked up at run time, or a network which can be traversed very simply. Alternatively, there may be no need to provide as powerful a mechanism as two-level morphology at all; a simpler device such as affix stripping (Alshawi, 1992, p119ff) or merely listing all inflected forms explicitly may be preferable.

For agglutinative languages such as Korean, Finnish and Turkish (Kwon and Karttunen, 1994; Koskenniemi, 1983; Oflazer, 1993), dimension (b) is very large, so creating an exhaustive word list is out of the question unless the lexicon is trivial. Compilation to a network may still make sense, however, and because these languages tend to exhibit few non-concatenative morphophonological phenomena other than vowel harmony, the continuation class mechanism may suffice to describe the allowed affix sequences at the surface level.

Many European languages are of the inflecting type, and occupy still another region of the space of difficulty. They are too complex morphologically to yield easily to the simpler techniques that can work for English. The phonological or orthographic changes involved in affixation may be quite complex, so dimension (a) can be large, and a feature mechanism may be needed to handle such varied but interrelated morphosyn- 
tactic phenomena such as umlaut (Trost, 1991), case, number, gender, and different morphological paradigms. On the other hand, while there may be many different affixes, their possibilities for combination within a word are fairly limited, so dimension (b) is quite manageable.

This paper describes a representation and associated compiler intended for two-level morphological descriptions of the written forms of inflecting languages. The system described is a component of the Core Language Engine (CLE; Alshawi, 1992), a general-purpose language analyser and generator implemented in Prolog which supports both a built-in lexicon and access to large external lexical databases. In this context, highly efficient word analysis and generation at run-time are less important than ensuring that the morphology mechanism is expressive, is easy to debug, and allows relatively quick compilation. Morphology also needs to be well integrated with other processing levels. In particular, it should be possible to specify relations among morphosyntactic and morphophonological rules and lexical entries; for the convenience of developers, this is done by means of feature equations. Further, it cannot be assumed that the lexicon has been fully specified when the morphology rules are compiled. Developers may wish to add and test further lexical entries without frequently recompiling the rules, and it may also be necessary to deal with unknown words at run time, for example by querying a large external lexical database or attempting spelling correction (Alshawi, 1992, pp124-7). Also, both analysis and generation of word forms are required. Run-time speed need only be enough to make the time spent on morphology small compared to sentential and contextual processing.

These parameters - languages with a complex morphology/syntax interface but a limited number of affix combinations, tasks where the lexicon is not necessarily known at compile time, bidirectional processing, and the need to ease development rather than optimize run-time efficiency dictate the design of the morphology compiler described in this paper, in which spelling rules and possible affix combinations (items (a) and (b)), but not the lexicon (item (c)), are composed in the compilation phase. Descriptions of French, Polish and English inflectional morphology have been developed for it, and I show how various aspects of the mechanism allow phenomena in these languages to be handled.

\section{The Description Language}

\subsection{Morphophonology}

The formalism for spelling rules (dimension (a)) is a syntactic variant of that of Ruessink (1989) and Pulman (1991). A rule is of the form
spell(Name, Surface Op Lexical, Classes, Features).

Rules may be optional ( $O p$ is " $\Rightarrow$ ") or obligatory (Op is " $\Leftrightarrow$ "). Surface and Lexical are both strings of the form

\section{"LContext $\mid$ Target $\mid$ RContext"}

meaning that the surface and lexical targets may correspond if the left and right contexts and the Features specification are satisfied. The vertical bars simply separate the parts of the string and do not themselves match letters. The correspondence between surface and lexical strings for an entire word is licensed if there is a partitioning of both so that each partition (pair of corresponding surface and lexical targets) is licensed by a rule, and no partition breaks an obligatory rule. A partition breaks an obligatory rule if the surface target does not match but everything else, including the feature specification, does.

The Features in a rule is a list of Feature $=$ Value equations. The allowed (finite) set of values of each feature must be prespecified. Value may be atomic or it may be a boolean expression.

Members of the surface and lexical strings may be characters or classes of single characters. The latter are represented by a single digit $N$ in the string and an item $N / C l a s s N a m e$ in the Classes list; multiple occurrences of the same $N$ in a single rule must all match the same character in a given application.

Figure 1 shows three of the French spelling rules developed for this system. The change_e_e 1 rule (simplified slightly here) makes it obligatory for a lexical $e$ to be realised as a surface $\dot{e}$ when followed by $t, r$, or $l$, then a morpheme boundary, then $e$, as long as the feature cdouble has an appro priate value. The default rule that copies characters between surface and lexical levels and the boundary rule that deletes boundary markers are both optional. Together these rules permit the following realization of cher ("expensive") followed by $e$ (feminine gender suffix) as chère, as shown in Figure 2. Because of the obligatory nature of change_e_e1, and the fact that the orthographic feature restriction on the root cher, [cdouble $=n$ ], is consistent with the one on that rule, an alternative realisation chere, involving the use of the default rule in third position, is ruled out. ${ }^{1}$

Unlike many other flavours of two-level morphology, the Target parts of a rule need not consist of a single character (or class occurrence); they can contain more than one, and the surface target may be empty. This obviates the need for "null" characters at the surface. However, although surface targets of any length can usefully be specified, it is in practice a good strategy

\footnotetext{
${ }^{1}$ The cdouble feature is in fact used to specify the spelling changes when $e$ is added to various stems cher $+\mathrm{e}=$ chère, achet $+\mathrm{e}=$ achète, but jet $+\mathrm{e}=$ jette .
} 


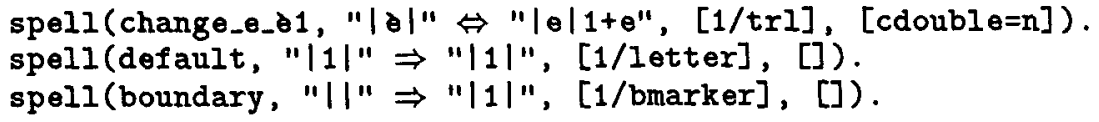

Figure 1: Three spelling rules

\begin{tabular}{|l|c|c|c|c|c|c|c|}
\hline Surface: & $\mathrm{c}$ & $\mathrm{h}$ & $\dot{\mathrm{e}}$ & $\mathrm{r}$ & $\mathrm{e}$ & \\
Lexical: & $\mathrm{c}$ & $\mathrm{h}$ & $\mathrm{e}$ & $\mathrm{r}$ & + & $\mathrm{e}$ & + \\
\hline Rule: & def. & $\mathrm{def}$. & $c_{.} e_{-} \dot{e} 1$ & $d e f$. & $b d y$ & $d e f$. & $b d y$. \\
\hline
\end{tabular}

Figure 2: Partitioning of chère as chertet

always to make lexical targets exactly one character long, because, by definition, an obligatory rule cannot block the application of another rule if their lexical targets are of different lengths. The example in Section 4.1 below clarifies this point.

\subsection{Word Formation and Interfacing to Syntax}

The allowed sequences of morphemes, and the syntactic and semantic properties of morphemes and of the words derived by combining them, are specified by morphosyntactic production rules (dimension (b)) and lexical entries both for affixes (dimension (b)) and for roots (dimension (c)), essentially as described by Alshawi (1992) (where the production rules are referred to as "morphology rules"). Affixes may appear explicitly in production rules or, like roots, they may be assigned complex feature-valued categories. Information, including the creation of logical forms, is passed between constituents in a rule by the sharing of variables. These feature-augmented production rules are just the same device as those used in the CLE's syntactico-semantic descriptions, and are a much more natural way to express morphotactic information than finite-state devices such as continuation classes (see Trost and Matiasek, 1994, for a related approach).

The syntactic and semantic production rules for deriving the feminine singular of a French adjective by suffixation with " $e$ " are given, with some details omitted, in Figure 3 . In this case, nearly all features are shared between the inflected word and the root, as is the logical form for the word (shown as Adj in the deriv rule). The only differing feature is that for gender, shown as the third argument of the eagr macro, which itself expands to a category.

Irregular forms, either complete words or affixable stems, are specified by listing the morphological rules and terminal morphemes from which the appropriate analyses may be constructed, for example:

$$
\begin{gathered}
\text { irreg(dit, [dire, 'PRESENT_3s'], } \\
\text { [v_v_affix-only]). }
\end{gathered}
$$

Here, PRESENT_3s is a pseudo-affix which has the same syntactic and semantic information attached to it as (one sense of) the affix " $t$ ", which is used to form some regular third person singulars. However, the spelling rules make no reference to PRESENT_3s; it is simply a device allowing categories and logical forms for irregular words to be built up using the same production rules as for regular words.

\section{Compilation}

All rules and lexical entries in the CLE are compiled to a form that allows normal Prolog unification to be used for category matching at run time. The same compiled forms are used for analysis and generation, but are indexed differently. Each feature for a major category is assigned a unique position in the compiled Prolog term, and features for which finite value sets have been specified are compiled into vectors in a form that allows boolean expressions, involving negation as well as conjunction and disjunction, to be conjoined by unification (see Mellish, 1988; Alshawi, 1992, pp46-48).

The compilation of morphological information is motivated by the nature of the task and of the languages to be handled. As discussed in Section 1 , we expect the number of affix combinations to be limited, but the lexicon is not necessarily known in advance. Morphophonological interactions may be quite complex, and the purpose of morphological processing is to derive syntactic and semantic analyses from words and vice versa for the purpose of full NLP. Reasonably quick compilation is required, and run-time speed need only be moderate.

\subsection{Compiling Spelling Patterns}

Compilation of individual spell rules is straightforward; feature specifications are compiled to positional/boolean format, characters and occurrences of character classes are also converted to boolean vectors, and left contexts are reversed ( $\mathrm{cf}$ Abrahamson, 1992) for efficiency. However, although it would be possible to analyse words directly with individually compiled rules (see Section 5 below), it can take an unacceptably long time to do so, largely because of the wide range of 


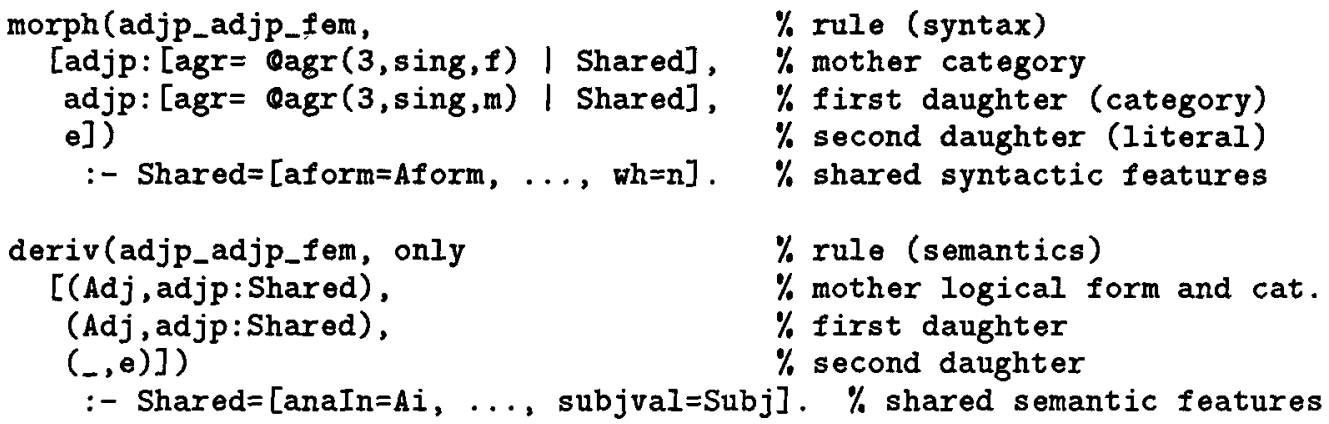

Figure 3: Syntactic and semantic morphological production rules

choices of rule available at each point and the need to check at each stage that obligatory rules have not been broken. We therefore take the following approach.

First, all legal sequences of morphemes are produced by top-down nondeterministic application of the production rules (Section 2.2), selecting affixes but keeping the root morpheme unspecified because, as explained above, the lexicon is undetermined at this stage. For example, for English, the sequences $*+e d+1 y$ and un+*+ing are among those produced, the asterisk representing the unspecified root.

Then, each sequence, together with any associated restrictions on orthographic features, undergoes analysis by the compiled spelling rules (Section 2.1), with the surface sequence and the root part of the lexical sequence initially uninstantiated. Rules are applied recursively and nondeterministically, somewhat in the style of Abramson (1992), taking advantage of Prolog's unification mechanism to instantiate the part of the surface string corresponding to affixes and to place some spelling constraints on the start and/or end of the surface and/or lexical forms of the root.

This process results in a set of spelling patterns, one for each distinct application of the spelling rules to each affix sequence suggested by the production rules. A spelling pattern consists of partially specified surface and lexical root character sequences; fully specified surface and lexical affix sequences, orthographic feature constraints associated with the spelling rules and affixes used, and a pair of syntactic category specifications derived from the production rules used. One category is for the root form, and one for the inflected form.

Spelling patterns are indexed according to the surface (for analysis) and lexical (for generation) affix characters they involve. At run time, an inflected word is analysed nondeterministically in several stages, each of which may succeed any number of times including zero.

- stripping off possible (surface) affix characters in the word and locating a spelling pattern that they index;
- matching the remaining characters in the word against the surface part of the spelling pattern, thereby, through shared variables, instantiating the characters for the lexical part to provide a possible root spelling;

- checking any orthographic feature constraints on that root;

- finding a lexical entry for the root, by any of a range of mechanisms including lookup in the system's own lexicon, querying an external lexical database, or attempting to guess an entry for an undefined word; and

- unifying the root lexical entry with the root category in the spelling pattern, thereby, through variable sharing with the other category in the pattern, creating a fully specified category for the inflected form that can be used in parsing.

In generation, the process works in reverse, starting from indexes on the lexical affix characters.

\subsection{Representing Lexical Roots}

Complications arise in spelling rule application from the fact that, at compile time, neither the lexical nor the surface form of the root, nor even its length, is known. It would be possible to hypothesize all sensible lengths and compile separate spelling patterns for each. However, this would lead to many times more patterns being produced than are really necessary.

Lexical (and, after instantiation, surface) strings for the unspecified roots are therefore represented in a more complex but less redundant way: as a structure

$$
L_{1} \ldots L_{m} \text { v }(L, R) R_{1} \ldots R_{n} .
$$

Here the $L_{i}$ 's are variables later instantiated to single characters at the beginning of the root, and $L$ is a variable, which is later instantiated to a list of characters, for its continuation. Similarly, the $R_{i}$ 's represent the end of the root, and $R$ is the continuation (this time reversed) leftwards into the root from the $R_{1}$. The $\mathrm{v}(L, R)$ structure is always matched specially with a Kleene-star of 
the default spelling rule. For full generality and minimal redundancy, $L_{m}$ and $R_{1}$ are constrained not to match the default rule, but the other $L_{i}$ 's and $R_{i}$ 's may. The values of $n$ required are those for which, for some spelling rule, there are $k$ characters in the target lexical string and $n-k$ from the beginning of the right context up to (but not including) a boundary symbol. The lexical string of that rule may then match $R_{1}, \ldots, R_{k}$, and its right context match $R_{k+1}, \ldots, R_{n},+, \ldots$. The required values of $m$ may be calculated similarly with reference to the left contexts of rules. ${ }^{2}$

During rule compilation, the spelling pattern that leads to the run-time analysis of chère given above is derived from $m=0$ and $n=2$ and the specified rule sequence, with the variables $R_{1} R_{2}$ matching as in Figure 4.

\subsection{Applying Obligatory Rules}

In the absence of a lexical string for the root, the correct treatment of obligatory rules is another problem for compilation. If an obligatory rule specifies that lexical $X$ must be realised as surface $Y$ when certain contextual and feature conditions hold, then a partitioning where $X$ is realised as something other than $Y$ is only allowed if one or more of those conditions is unsatisfied. Because of the use of boolean vectors for both features and characters, it is quite possible to constrain each partitioning by unifying it with the complement of one of the conditions of each applicable obligatory rule, thereby preventing that rule from applying. For English, with its relatively simple inflectional spelling changes, this works well. However, for other languages, including French, it leads to excessive numbers of spelling patterns, because there are many obligatory rules with non-trivial contexts and feature specifications.

For this reason, complement unification is not actually carried out at compile time. Instead, the spelling patterns are augmented with the fact that certain conditions on certain obligatory rules need to be checked on certain parts of the partitioning when it is fully instantiated. This slows down runtime performance a little but, as we will see below, the speed is still quite acceptable.

\subsection{Timings}

The compilation process for the entire rule set takes just over a minute for a fairly thorough de-

\footnotetext{
${ }^{2}$ Alternations in the middle of a root, such as umlaut, can be handled straightforwardly by altering the root/affix pattern from $L_{1} \ldots L_{m} v(L, R) R_{1} \ldots R_{n}$ to $L_{1} \ldots L_{m}$ v $(L, R) M$ v $\left(L^{\prime}, R^{\prime}\right) R_{1} \ldots R_{n}$, with $M$ forbidden to be the default rule. This has not been necessary for the descriptions developed so far, but its implementation is not expected to lead to any great decrease in run-time performance, because the nondeterminism it induces in the lookup process is no different in kind from that arising from alternations at root-affix boundaries.
}

scription of French inflectional morphology, running on a Sparcstation 10/41 (SPECint92 $=52.6$ ). Run-time speeds are quite adequate for full NLP, and reflect the fact that the system is implemented in Prolog rather than (say) $\mathrm{C}$ and that full syntactico-semantic analyses of sentences, rather than just morpheme sequences or acceptability judgments, are produced.

Analysis of French words using this rule set and only an in-core lexicon averages around 50 words per second, with a mean of 11 spelling analyses per word leading to a mean of 1.6 morphological analyses (the reduction being because many of the roots suggested by spelling analysis do not exist or cannot combine with the affixes produced). If results are cached, subsequent attempts to analyse the same word are around 40 times faster still. Generation is also quite acceptably fast, running at around 100 words per second; it is slightly faster than analysis because only one spelling, rather than all possible analyses, is sought from each call. Because of the separation between lexical and morphological representations, these timings are essentially unaffected by in-core lexicon size, as full advantage is taken of Prolog's built-in indexing.

Development times are at least as important as computation times. A rule set embodying a quite comprehensive treatment of French inflectional morphology was developed in about one person month. The English spelling rule set was adapted from Ritchie et al (1992) in only a day or two. A Polish rule set is also under development, and Swedish is planned for the near future.

\section{Some Examples}

To clarify further the use of the formalism and the operation of the mechanisms, we now examine several further examples.

\subsection{Multiple-letter spelling changes}

Some obligatory spelling changes in French involve more than one letter. For example, masculine adjectives and nouns ending in eau have feminine counterparts ending in elle: beau ("nice") becomes belle, chameau ("camel") becomes chamelle. The final $e$ is a feminizing affix and can be seen as inducing the obligatory spelling change $a u \rightarrow l l$. However, although the obvious spelling rule,

$$
\text { speII (change_au_II, "|II|" } \leftrightarrow \text { "|au } \mid+e^{\prime)} \text {, }
$$

allows this change, it does not rule out the incorrect realization of beau+e as *beaue, shown in Figure 5 , because it only affects partitionings where the au at the lexical level forms a single partition, rather than one for a and one for $u$. Instead, the following pair of rules, in which the lexical targets have only one character each, achieve the desired effect: 


\begin{tabular}{|c|c|c|c|c|c|c|c|c|}
\hline \multirow{2}{*}{$\begin{array}{l}\text { Compile } \\
\text { time: }\end{array}$} & Rule: & \multicolumn{2}{|c|}{ def." } & \multirow{2}{*}{$\frac{c . e_{-} \grave{e} 1}{R_{1}}$} & \multirow{2}{*}{$\frac{d e f}{R_{2}}$} & $b d y$. & def. & $b d y$. \\
\hline & Variable: & & $R)$ & & & \multicolumn{3}{|c|}{$\ldots$} \\
\hline \multirow{2}{*}{$\begin{array}{l}\text { Run } \\
\text { time: }\end{array}$} & Surface: & c & $\mathrm{h}$ & $\ddot{\mathrm{e}}$ & $\mathrm{r}$ & & e & \\
\hline & Lexical: & $\mathrm{c}$ & h & $\mathrm{e}$ & $\mathrm{r}$ & $t$ & $\mathrm{e}$ & + \\
\hline
\end{tabular}

Figure 4: Spelling pattern application to the analysis of chère

\begin{tabular}{|l|c|c|c|c|c|c|c|}
\hline Surface: & $\mathrm{b}$ & $\mathrm{e}$ & $\mathrm{a}$ & $\mathrm{u}$ & $\mathrm{e}$ & \\
Lexical: & $\mathrm{b}$ & $\mathrm{e}$ & $\mathrm{a}$ & $\mathrm{u}$ & + & $\mathrm{e}$ & + \\
\hline Rule: & def. & def. & def. & def. & bdy. & def. & bdy. \\
\hline
\end{tabular}

Figure 5: Incorrect partitioning for beaute+

spel1 (change_au_111, "|1|"↔" $|a| u+e ")$ spel1 (change_au_112, "|1|"↔"a|u|+e")

Here, change_au_111 rules out a:a partition in Figure 5, and change_au_112 rules out the $u: u$ one.

It is not necessary for the surface target to contain exactly one character for the blocking effect to apply, because the semantics of obligatoriness is that the lexical target and all contexts, taken together, make the specified surface target (of whatever length) obligatory for that partition. The reverse constraint, on the lexical target, does not apply.

\subsection{Using features to control rule application}

Features can be used to control the application of rules to particular lexical items where the applicability cannot be deduced from spellings alone. For example, Polish nouns with stems whose final syllable has vowel $\delta$ normally have inflected forms in which the accent is dropped. Thus in the nominative plural, krój ("style") becomes kroje, bór ("forest") becornes bory, bój ("combat") becomes boje. However, there are exceptions, such as zbój ("bandit") becoming zbóje. Similarly, some French verbs whose infinitives end in -eler take a grave accent on the first $e$ in the third person singular future (modeler, "model", becomes modèlera), while others double the $l$ instead (e.g. appeler, "call", becomes appellera).

These phenomena can be handled by providing an obligatory rule for the case whether the letter changes, but constraining the applicability of the rule with a feature and making the feature clash with that for roots where the change does not occur. In the Polish case:

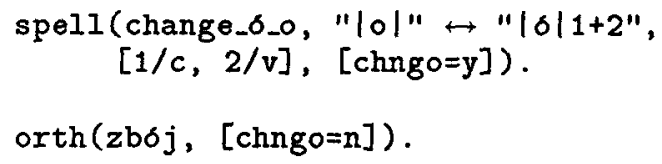

Then the partitionings given in Figure 6 will be the only possible ones. For bój, the change_o_o rule must apply, because the chngo feature for $b 6 j$ is unspecified and therefore can take any value; for $z b o ́ j$, however, the rule is prevented from applying by the feature clash, and so the default rule is the only one that can apply.

\section{Debugging the Rules}

The debugging tools help in checking the operation of the spelling rules, either (1) in conjunction with other constraints or (2) on their own.

For case (1), the user may ask to see all inflections of a root licensed by the spelling rules, production rules, and lexicon; for cher, the output is

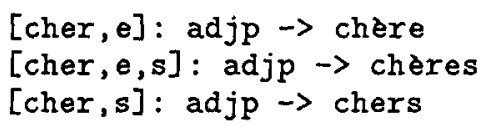

meaning that when cher is an adjp (adjective) it may combine with the suffixes listed to produce the inflected forms shown. This is useful in checking over- and undergeneration. It is also possible to view the spelling patterns and production rule tree used to produce a form; for chère, the trace (slightly simplified here) is as in figure 7 . The spelling pattern 194 referred to here is the one depicted in a different form in Figure 4. The notation \{clmnprstv=A\} denotes a set of possible consonants represented by the variable $A$, which also occurs on the right hand side of the rule, indicating that the same selection must be made for both occurrences. Production rule tree 17 is that for a single application of the rule adjp_adjp_f em, which describes the feminine form of the an adjective, where the root is taken to be the masculine form. The Root and Infl lines show the features that differ between the root and inflected forms, while the Both line shows those that they share. Tree 18, which is also pointed to by the spelling pattern, describes the feminine forms of nouns analogously.

For case (2), the spelling rules may be applied directly, just as in rule compilation, to a specified surface or lexical character sequence, as if no 


\begin{tabular}{|c|c|c|c|c|c|c|}
\hline $\begin{array}{l}\text { Surface: } \\
\text { Lexical: }\end{array}$ & $\begin{array}{l}b \\
b\end{array}$ & $\begin{array}{l}\text { O } \\
\text { ó }\end{array}$ & $\begin{array}{l}j \\
j\end{array}$ & + & $\begin{array}{l}\mathrm{e} \\
\mathrm{e}\end{array}$ & + \\
\hline Rule: & def. & $c_{-} \delta_{-} o$. & def & $b d y$. & def. & $b d y$. \\
\hline
\end{tabular}

\begin{tabular}{|l|c|c|c|c|c|c|c|}
\hline Surface: & $\mathrm{z}$ & $\mathrm{b}$ & $\mathrm{o}$ & $\mathrm{j}$ & & $\mathrm{e}$ & \\
Lexical: & $\mathrm{z}$ & $\mathrm{b}$ & $\mathrm{o}$ & $\mathrm{j}$ & + & $\mathrm{e}$ & + \\
\hline Rule: & def. & def. & def. & def. & bdy. & def. & $b d y$. \\
\hline
\end{tabular}

Figure 6: Feature-dependent dropping of accent

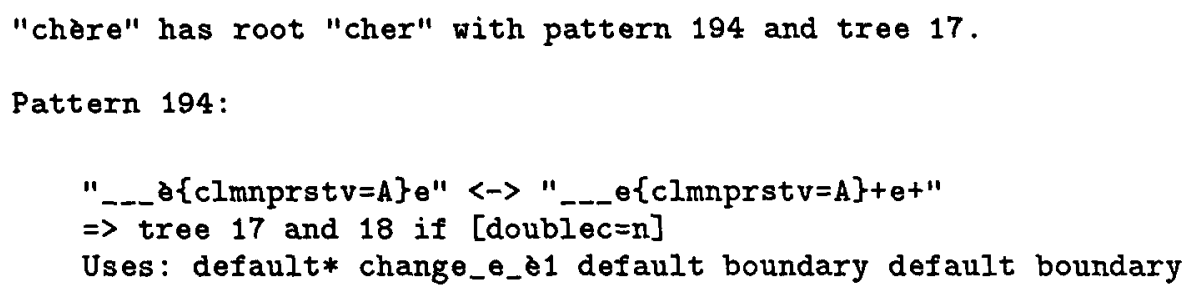

Tree 17:

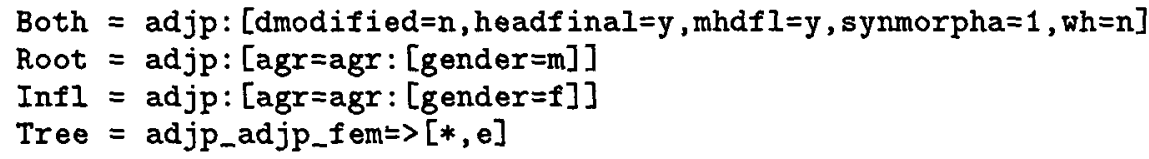

Figure 7: Debugger trace of derivation of chère

lexical or morphotactic constraints existed. Feature constraints, and cases where the rules will not apply if those constraints are broken, are shown. For the lexical sequence cher+et, for example, the output is as follows.

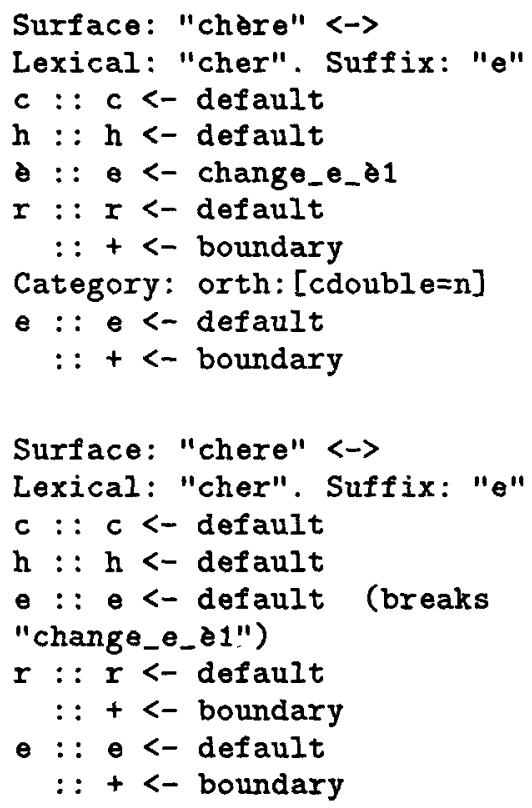

This indicates to the user that if cher is given a lexical entry consistent with the constraint cdouble $=n$, then only the first analysis will be valid; otherwise, only the second will be.

\section{Conclusions and Further Work}

The rule formalism and compiler described here work well for European languages with reasonably complex orthographic changes but a limited range of possible affix combinations. Development, compilation and run-time efficiency are quite acceptable, and the use of rules containing complex feature-augmented categories allows morphotactic behaviours and non-segmental spelling constraints to be specified in a way that is perspicuous to linguists, leading to rapid development of descriptions adequate for full NLP.

The kinds of non-linear effects common in Semitic languages, where vowel and consonant patterns are interpolated in words (Kay, 1987; Kiraz, 1994) could be treated efficiently by the mechanisms described here if it proved possible to define a representation that allowed the parts of an inflected word corresponding to the root to be separated fairly cleanly from the parts expressing the inflection. The latter could then be used by a modified version of the current system as the basis for efficient lookup of spelling patterns which, as in the current system, would allow possible lexical roots to be calculated.

Agglutinative languages could be handled ef- 
ficiently by the current mechanism if specifications were provided for the affix combinations that were likely to occur at all often in real texts. A backup mechanism could then be provided which attempted a slower, but more complete, direct application of the rules for the rarer cases.

The interaction of morphological analysis with spelling correction (Carter, 1992; Oflazer, 1994; Bowden, 1995) is another possibly fruitful area of work. Once the root spelling patterns and the affix combinations pointing to them have been created, analysis essentially reduces to an instance of affixstripping, which would be amenable to exactly the technique outlined by Carter (1992). As in that work, a discrimination net of root forms would be required; however, this could be augmented independently of spelling pattern creation, so that the flexibility resulting from not composing the lexicon with the spelling rules would not be lost.

\section{Acknowledgments}

I am grateful to Manny Rayner and anonymous European ACL referees for commenting on earlier versions of this paper, and to Pierrette Bouillion and Matgorzata Styś for comments and also for providing me with their analyses of the French and Polish examples respectively.

This research was partly funded by the Defence Research Agency, Malvern, UK, under Strategic Research Project M2YBT44X.

\section{References}

Abramson, H., (1992). "A Logic Programming View of Relational Morphology". Proceedings of COLING-92, 850-854.

Alshawi, H. (1992). The Core Language Engine (ed). MIT Press.

Alshawi, H., D.J. Arnold, R. Backofen, D.M. Carter, J. Lindop, K. Netter, S.G. Pulman, J. Tsujii, and H. Uszkoreit (1991). Eurotra ET6/1: Rule Formalism and Virtual Machine Design Study. Commission of the European Communities, Luxembourg.

Bowden, T. (1995) "Cooperative Error Handling and Shallow Processing", these proceedings.

Carter, D.M. (1992). "Lattice-based Word Identification in CLARE". Proceedings of ACL-92.

Kaplan, R., and M. Kay (1994). "Regular Models of Phonological Rule Systems", Computational Linguistics, 20:3, 331-378.

Kay, M. (1987). "Non-concatenative Finite-State Morphology". Proceedings of EACL-87.

Karttunen, L., R.M. Kaplan, and A. Zaenen (1992). "Two-level Morphology with Composition". Proceedings of COLING-92, 141148.
Kiraz, G. (1994). "Multi-tape Two-level Morphology". Proceedings of COLING-94, 180-186.

Koskenniemi, K. (1983). Two-level morphology: a general computational model for word-form recognition and production. University of Helsinki, Department of General Linguistics, Publications, No. 11.

Kwon, H-C., and L. Karttunen (1994). "Incremental Construction of a Lexical Transducer for Korean". Proceedings of COLING-94, 1262-1266

Mellish, C. S. (1988). "Implementing Systemic Classification by Unification". Computational Linguistics 14:40-51.

Oflazer, K. (1993). "Two-level Description of Turkish Morphology". Proceedings of European $A C L-93$.

Oflazer, K. (1994). Spelling Correction in Agglutinative Languages. Article 9410004 in cmp-lg@xxx. lanl.gov archive.

Ritchie, G., G.J. Russell, A.W. Black and S.G. Pulman (1992). Computational Morphology. MIT Press.

Ruessink, H. (1989). Two Level Formalisms. Utrecht Working Papers in NLP, no. 5.

Trost, H. (1990). "The Application of Two-level Morphology to Non-Concatenative German Morphology". Proceedings of COLING-90, 371-376.

Trost, H. (1991). "X2MORF: A Morphological Component Based on Augmented Twolevel Morphology". Proceedings of IJCAI-91, 1024-1030.

Trost, H., and J. Matiasek (1994). "Morphology with a Null-Interface", Proceedings of COLING-94. 